\title{
CFT and Logarithmic Corrections to the Black Hole Entropy Product Formula
}

\author{
Parthapratim Pradhan \\ Department of Physics, Hiralal Mazumdar Memorial College for Women, Dakshineswar, Kolkata 700035, India \\ Correspondence should be addressed to Parthapratim Pradhan; pppradhan77@gmail.com
}

Received 28 March 2017; Accepted 23 May 2017; Published 30 July 2017

Academic Editor: Elias C. Vagenas

Copyright (C) 2017 Parthapratim Pradhan. This is an open access article distributed under the Creative Commons Attribution License, which permits unrestricted use, distribution, and reproduction in any medium, provided the original work is properly cited. The publication of this article was funded by SCOAP ${ }^{3}$.

\begin{abstract}
We examine the logarithmic corrections to the black hole $(\mathrm{BH})$ entropy product formula of outer horizon and inner horizon by taking into account the effects of statistical quantum fluctuations around the thermal equilibrium and via conformal field theory (CFT). We argue that, in logarithmic corrections to the BH entropy product formula when calculated using CFT and taking into account the effects of quantum fluctuations around the thermal equilibrium, the formula should not be universal and it also should not be quantized. These results have been explicitly checked by giving several examples.
\end{abstract}

\section{Introduction}

There has been considerable ongoing excitement in physics of $\mathrm{BH}$ thermodynamic product formula [particularly area (or entropy) product formula] of inner horizon $\left(\mathscr{H}^{-}\right)$and outer horizon $\left(\mathscr{H}^{+}\right)$for a wide variety of BHs [1-9] which have been examined so far without considering any logarithmic correction. For several cases, the product is mass-independent (universal) and in some specific cases the product is not massindependent. This investigation has been more sparked by Cvetič et al. [2] for supersymmetric BPS (Bogomol'ni-PrasadSommerfield) class of BHs which have inner and outer $\mathrm{BH}$ entropy of the form $\delta_{ \pm}=2 \pi\left(\sqrt{N_{L}} \pm \sqrt{N_{R}}\right)$, where $N_{L}$ and $N_{R}$ are excitation numbers of the left and right moving sectors of a weakly coupled two-dimensional (2D) CFT. Therefore, their product $\delta_{+} \mathcal{\delta}_{-}$must be quantized in nature.

It has been suggested by Larsen [10] that $\mathrm{BH}$ event horizon is quantized in Planck units so it is natural to be valid for Cauchy horizon also. This also indicates that the product of area (or entropy) is quantized in terms of quantized charges and quantized angular momentum and so on. But this has been discussed without considering any logarithmic correction to the $\mathrm{BH}$ entropy. Now if we take into account the logarithmic correction to the $\mathrm{BH}$ entropy, what happens in case of logarithmic correction to the $\mathrm{BH}$ entropy product formula? What are the quantization rules for logarithmic corrected $\mathrm{BH}$ entropy product formula? These are the main issues that we will be discussed in this work.

It should be noted that this is the continuation of our earlier investigation [11]. In the previous work, we derived the general logarithmic correction to the entropy product formula of event horizon and Cauchy horizon for various spherically symmetric and axisymmetric $\mathrm{BHs}$ by taking into account the effects of quantum fluctuations around the thermal equilibrium. These corrections are evaluated in terms of some $\mathrm{BH}$ thermodynamic parameters, namely, the specific heat $C_{+}$of $\mathscr{H}^{ \pm}$and $B H$ temperature $T_{+}$of $\mathscr{H}^{ \pm}$, respectively.

The logarithmic correction of $\mathrm{BH}$ entropy of $\mathscr{H}^{ \pm}$is described by the key formula

$$
\mathcal{S}_{ \pm}=\ln \rho_{ \pm}=\mathcal{S}_{0, \pm}-\frac{1}{2} \ln \left|C_{ \pm} T_{ \pm}^{2}\right|+\cdots,
$$

where $\rho_{ \pm}$is density of states of $\mathscr{H}^{ \pm}$and $\mathcal{S}_{ \pm}$is entropy of $\mathscr{H}^{ \pm}$ and their product is derived to be

$$
\begin{aligned}
\mathcal{S}_{+} \mathcal{S}_{-}= & \mathcal{S}_{0,+} \mathcal{S}_{0,-} \\
& -\frac{1}{2}\left[\mathcal{S}_{0,+} \ln \left|C_{-} T_{-}^{2}\right|+\mathcal{S}_{0,-} \ln \left|C_{+} T_{+}^{2}\right|\right] \\
& +\frac{1}{4} \ln \left|C_{+} T_{+}^{2}\right| \ln \left|C_{-} T_{-}^{2}\right|+\cdots,
\end{aligned}
$$


where $\mathcal{S}_{0, \pm}$ is the entropy of $\mathscr{H}^{ \pm}$without logarithmic correction.

In the present work, we shall compute the general logarithmic correction to BH entropy of $\mathscr{H}^{ \pm}$whenever we have taken the effects of statistical quantum fluctuations around the thermal equilibrium and by using exact entropy function $\delta_{ \pm}\left(\beta_{ \pm}\right)$according to the formalism borrowed from the quantum theory of gravity [12-25]. Whenever we incorporated the effects of quantum fluctuations around the thermal equilibrium, the Bekenstein-Hawking entropy formula must be corrected and the entropy product formula of $\mathscr{H}^{ \pm}$must also be corrected. This is the main motivation behind this work.

It has been known that BHs in Einstein's gravity as well as other theories of gravity are much larger than the Planck scale length where the Bekenstein-Hawking entropy is precisely proportional to the horizon area $[12,13,18-20]$. Thus, it is quite natural to investigate the leading order corrections in Bekenstein-Hawking entropy as well as in Bekenstein-Hawking entropy product formula of $\mathscr{H}^{ \pm}$and when one can reduce the size of $\mathrm{BH}$. For large $\mathrm{BHs}$, it has been proved that the logarithm of the density of states is exactly the BekensteinHawking entropy plus the corrections term $-\chi \ln A$, where $\chi=3 / 2$ and $A$ is the area of the event horizon [22, 23]. Thus, it appears that the logarithmic corrections to the Bekenstein-Hawking entropy as well as Bekenstein-Hawking entropy product formula of $\mathscr{H}^{ \pm}$are a generic feature of BHs. It has been verified earlier by Das et al. [24] for $\mathscr{H}^{+}$and here we have tried to examine for $\mathscr{H}^{-}$followed by our earlier investigation [11].

It should be emphasized that logarithmic corrections to the Bekenstein-Hawking formula are very interesting and a great deal about such corrections is known in string theory and beyond. Logarithmic corrections arise from various sources, the simplest of which are the statistical fluctuations around thermal equilibrium. These are always present because they arise from saddle point Gaussian corrections to the integral that computes the density of states from the partition function. In some cases, such as the BTZ BH in pure 3D gravity, these are the only logarithmic corrections to the Bekenstein-Hawking entropy. However, more generally the logarithm of the partition function, $\ln Z$, itself receives corrections from the massless spectrum of particles in the theory whose solution contains the $\mathrm{BH}$. These corrections therefore cannot be determined from the BH solution only. They are universal only in the sense that they are independent from the UV completion of the theory (see review article [26]).

Moreover, it must be noted that a given theory of quantum gravity will assign a Hilbert space to $\mathscr{H}^{+}$counting the number of microstates of the Hilbert space which gives us the entropy of $\mathscr{H}^{+}$of the BH by Boltzmann's entropy formula

$$
\mathcal{S}_{+}=k_{B} \ln \Omega_{+},
$$

where $k_{B}$ is the Boltzmann constant and $\Omega_{+}$is the microstates of $\mathscr{H}^{+}$only. Analogously, there must exists inner Hilbert space for $\mathscr{H}^{-}$. Therefore, Boltzmann's entropy formula for $\mathscr{H}^{-}$ becomes

$$
\mathcal{\delta}_{-}=k_{B} \ln \Omega_{-},
$$

where $\Omega_{-}$is the microstates of $\mathscr{H}^{-}$. Finally, their product should be

$$
\mathcal{S}_{+} \mathcal{S}_{-}=k_{B}^{2} \ln \Omega_{+} \ln \Omega_{-} .
$$

Now we turn to compute the logarithmic corrections to $\mathrm{BH}$ entropy product formula by using the CFT formalism.

\section{Logarithmic Corrections to the BH Entropy Product Formula of $\mathscr{H}^{ \pm}$via CFT}

We have started with the partition function [11, 24] of any thermodynamic system consisting of $\mathscr{H}^{ \pm}$which should read

$$
\mathscr{Z}_{ \pm}\left(\beta_{ \pm}\right)=\int_{0}^{\infty} \rho_{ \pm}(E) e^{-\beta_{ \pm} E} d E
$$

where $T_{ \pm}=1 / \beta_{ \pm}$can be defined as the temperature of $\mathscr{H}^{ \pm}$. We have to set Boltzmann constant $k_{B}=1$.

The density of states of the said thermodynamic system may be expressed by taking an inverse Laplace transformation (keeping $E$ fixed) of the partition function defined at $\mathscr{H}^{ \pm}$ $[24,27,28]$

$$
\begin{aligned}
\rho_{ \pm}(E) & =\frac{1}{2 \pi i} \int_{c-i \infty}^{c+i \infty} \mathscr{Z}_{ \pm}\left(\beta_{ \pm}\right) e^{\beta_{ \pm} E} d \beta_{ \pm} \\
& =\frac{1}{2 \pi i} \int_{c-i \infty}^{c+i \infty} e^{S_{ \pm}\left(\beta_{ \pm}\right)} d \beta_{ \pm},
\end{aligned}
$$

where $c$ is a real constant and defining

$$
S_{ \pm}=\ln \mathscr{Z}_{ \pm}+\beta_{ \pm} E
$$

is the exact entropy of $\mathscr{H}^{ \pm}$as a function of temperature.

Now if we consider the system to be in equilibrium, then the inverse temperature is defined to be $\beta_{ \pm}=\beta_{0, \pm}$; therefore, we can expand the entropy function of $\mathscr{H}^{ \pm}$as

$$
S_{ \pm}\left(\beta_{ \pm}\right)=S_{0, \pm}+\frac{1}{2}\left(\beta_{ \pm}-\beta_{0, \pm}\right)^{2} S_{0, \pm}^{\prime \prime}+\cdots,
$$

where $S_{0, \pm}:=S_{ \pm}\left(\beta_{0, \pm}\right)$ and $S_{0, \pm}^{\prime \prime}=\partial^{2} S_{ \pm} / \partial \beta_{ \pm}^{2}$ at $\beta_{ \pm}=\beta_{0, \pm}$.

Substituting (9) in (7), we find

$$
\rho_{ \pm}(E)=\frac{e^{S_{0, \pm}}}{2 \pi i} \int_{c-i \infty}^{c+i \infty} e^{\left(\beta_{ \pm}-\beta_{0, \pm}\right)^{2} S_{0, \pm}^{\prime \prime} / 2} d \beta_{ \pm} .
$$

Let us put $\beta_{ \pm}-\beta_{0, \pm}=i x_{ \pm}$and choosing $c=\beta_{0, \pm}, x_{ \pm}$is a real variable and evaluating a contour integration, one obtains

$$
\rho_{ \pm}(E)=\frac{e^{S_{0, \pm}}}{\sqrt{2 \pi S_{0, \pm}^{\prime \prime}}} .
$$

Thus, the logarithm of the density of states gives the corrected entropy of $\mathscr{H}^{ \pm}$:

$$
\mathcal{S}_{ \pm}:=\ln \rho_{ \pm}=\mathcal{S}_{0, \pm}-\frac{1}{2} \ln S_{0, \pm}^{\prime \prime}+\cdots .
$$


The main aim of this work is to compute the term $S_{0, \pm}^{\prime \prime}$ by using the exact entropy function $\mathcal{S}_{ \pm}\left(\beta_{ \pm}\right)$, evaluated at the equilibrium temperature $\beta_{ \pm}=\beta_{0, \pm}$.

Assume that the exact entropy function $\mathcal{S}_{ \pm}\left(\beta_{ \pm}\right)$of $\mathscr{H}^{ \pm}$ (the entropy function is defined in [20] for $\mathscr{H}^{+}$only. We here prescribed that this entropy function is valid for $\mathscr{H}^{-}$as well) which is followed from the CFT [24] is

$$
\delta_{ \pm}\left(\beta_{ \pm}\right)=a \beta_{ \pm}+\frac{b}{\beta_{ \pm}} .
$$

We choose the entropy function [24] in more general form (because this form admits a saddle point) as

$$
\mathcal{S}_{ \pm}\left(\beta_{ \pm}\right)=a \beta_{ \pm}^{m}+\frac{b}{\beta_{ \pm}^{n}},
$$

where $m, n, a, b>0$. When we have considered the special case which is dictated by CFT, then $m=n=1$.

Now the above function has an extremum value at

$$
\beta_{0, \pm}=\left(\frac{n b}{m a}\right)^{1 /(m+n)} \equiv \frac{1}{T_{ \pm}} .
$$

Expanding around $\beta_{0, \pm}$ and by evaluating second-order derivative, one obtains

$$
\begin{aligned}
\mathcal{S}_{ \pm}\left(\beta_{ \pm}\right)= & \alpha\left(a^{n} b^{m}\right)^{1 /(m+n)} \\
& +\frac{\gamma}{2}\left(a^{n+2} b^{m-2}\right)^{1 /(m+n)}\left(\beta_{ \pm}-\beta_{0, \pm}\right)^{2}+\cdots,
\end{aligned}
$$

where

$$
\begin{aligned}
& \alpha=\left(\frac{n}{m}\right)^{m /(m+n)}+\left(\frac{m}{n}\right)^{n /(m+n)}, \\
& \gamma=(m+n) m^{((n+2) /(m+n))}+n^{((m-2) /(m+n))} .
\end{aligned}
$$

We also derived in [11] close to the equilibrium and at the inverse temperature $\beta_{ \pm}=\beta_{0, \pm}$ the entropy function of $\mathscr{H}^{ \pm}$ as

$$
S_{ \pm}\left(\beta_{ \pm}\right)=S_{0, \pm}+\frac{1}{2}\left(\beta_{ \pm}-\beta_{0, \pm}\right)^{2} S_{0, \pm}^{\prime \prime}+\cdots,
$$

where $S_{0, \pm}:=S_{ \pm}\left(\beta_{0, \pm}\right)$ and $S_{0, \pm}^{\prime \prime}=\partial^{2} S_{ \pm}\left(\beta_{ \pm}\right) / \partial \beta_{ \pm}^{2}$ at $\beta_{ \pm}=\beta_{0, \pm}$. Comparing (16) and (18), we find

$$
\begin{aligned}
& S_{0, \pm}=\alpha\left(a^{n} b^{m}\right)^{1 /(m+n)}, \\
& S_{0, \pm}^{\prime \prime}=\gamma\left(a^{n+2} b^{m-2}\right)^{1 /(m+n)} .
\end{aligned}
$$

Inverting these equations, one can find $a$ and $b$ in terms of $S_{0, \pm}$ and $S_{0, \pm}^{\prime \prime}$

$$
\begin{aligned}
& a=\left(\frac{\alpha^{(m-2) / 2}}{\gamma^{m / 2}}\right)\left[\frac{\left(S_{0, \pm}^{\prime \prime}\right)^{m / 2}}{\left(S_{0, \pm}\right)^{m / 2}}\right], \\
& b=\left[\frac{\gamma}{\alpha^{(n+2) / 2}}\right]^{n / 2}\left[\frac{\left(S_{0, \pm}^{\prime \prime}\right)^{(n+2) / 2}}{\left(S_{0, \pm}\right)^{n / 2}}\right] .
\end{aligned}
$$

Putting these values in (15), we get

$$
\beta_{0, \pm}=\frac{1}{T_{ \pm}}=\left(\frac{n}{m}\right)^{1 /(m+n)} \sqrt{\frac{\gamma S_{0, \pm}}{\alpha S_{0, \pm}^{\prime \prime}}} .
$$

Now inverting $S_{0, \pm}^{\prime \prime}$ in terms of $S_{0, \pm}$ and $T_{ \pm}$, one obtains

$$
S_{0, \pm}^{\prime \prime}=\left[\left(\frac{\gamma}{\alpha}\right)\left(\frac{n}{m}\right)^{2 /(m+n)}\right] S_{0, \pm} T_{ \pm}^{2} .
$$

Substituting these values in (12), we have

$$
\mathcal{S}_{ \pm}=\ln \rho_{ \pm}=\mathcal{S}_{0, \pm}-\frac{1}{2} \ln \left|T_{ \pm}^{2} S_{0, \pm}\right|+\cdots .
$$

This is in fact the generic formula for leading order correction to Bekenstein-Hawking formula. It should be noted that the formula is indeed independent of $a$ and $b$. What is new in this formula is that one could calculate the logarithmic correction to the Bekenstein-Hawking entropy of $\mathscr{H}^{ \pm}$without knowing the values of any specific heat of the BH but only knowing the values of $T_{ \pm}$of $\mathscr{H}^{ \pm}$and $S_{0, \pm}$ for the said BH.

Therefore, the product becomes

$$
\begin{aligned}
\mathcal{S}_{+} \mathcal{\delta}_{-}= & \mathcal{S}_{0,+} \mathcal{\delta}_{0,-} \\
& -\frac{1}{2}\left[\mathcal{S}_{0,+} \ln \left|T_{-}^{2} \mathcal{\delta}_{0,-}\right|+\mathcal{S}_{0,-} \ln \left|T_{+}^{2} \mathcal{\delta}_{0,+}\right|\right] \\
& +\frac{1}{4} \ln \left|T_{+}^{2} \mathcal{S}_{0,+}\right| \ln \left|T_{-}^{2} \mathcal{\delta}_{0,-}\right|+\cdots
\end{aligned}
$$

We have already argued the implication of this formula in [11] as when we take the first-order correction, it indicates that the product is always dependent on mass parameter. Therefore, the theorem of Ansorg-Hennig [1], "the area product formula of $\mathscr{H}^{ \pm}$being independent of mass," is no longer true when we have taken into consideration the leading order logarithmic correction.

For completeness, we further compute the logarithmic correction of entropy sum, entropy minus, and entropy division using (23). They are

$$
\begin{aligned}
\mathcal{S}_{+}+\mathcal{S}_{-}= & \ln \rho_{+}+\ln \rho_{-} \\
= & \left(\mathcal{S}_{0,+}+\mathcal{S}_{0,-}\right) \\
& -\frac{1}{2}\left[\ln \left|T_{+}^{2} \mathcal{S}_{0,+}\right|+\ln \left|T_{-}^{2} \mathcal{S}_{0,-}\right|\right]+\cdots \\
\mathcal{S}_{+}-\mathcal{S}_{-}= & \ln \rho_{+}-\ln \rho_{-} \\
= & \left(\mathcal{S}_{0,+}-\mathcal{S}_{0,-}\right) \\
& -\frac{1}{2}\left[\ln \left|T_{+}^{2} \mathcal{S}_{0,+}\right|-\ln \left|T_{-}^{2} \mathcal{S}_{0,-}\right|\right]+\cdots, \\
\frac{\mathcal{S}_{+}}{\mathcal{S}_{-}}= & \frac{\ln \rho_{+}}{\ln \rho_{-}}=\frac{\mathcal{S}_{0,+}-(1 / 2) \ln \left|T_{+}^{2} S_{0,+}\right|+\cdots}{\mathcal{S}_{0,-}-(1 / 2) \ln \left|T_{-}^{2} S_{0,-}\right|+\cdots} .
\end{aligned}
$$

These equations indicate that they are all mass-independent; hence, they are not universal in this sense. 


\section{Examples}

Now we apply this formula for specific BHs in order to calculate the logarithm correction to the Bekenstein-Hawking entropy of $\mathscr{H}^{ \pm}$. First we take the four-dimensional ReissnerNordstrøm (RN) BH.

Example 1 (RN BH). The $\mathrm{BH}$ entropy and $\mathrm{BH}$ temperature [15] become

$$
\begin{aligned}
\mathcal{S}_{0, \pm} & =\pi r_{ \pm}^{2}, \\
T_{ \pm} & =\frac{r_{ \pm}-r_{\mp}}{4 \pi r_{ \pm}^{2}},
\end{aligned}
$$

where $r_{ \pm}=M \pm \sqrt{M^{2}-Q^{2}}$ and $M$ and $Q$ are mass and charge of $\mathrm{BH}$, respectively.

Therefore, the entropy correction is given by

$$
\delta_{ \pm}=\ln \rho_{ \pm}=\delta_{0, \pm}-\frac{1}{2} \ln \left|\frac{\left(r_{ \pm}-r_{\mp}\right)^{2}}{16 \pi r_{ \pm}^{2}}\right|+\cdots .
$$

We can conclude that the product includes the mass term so it is not universal.

The second example we take is the Kehagias-Sfetsos (KS) $\mathrm{BH}$ [29] in Hořava-Lifshitz gravity [30-32].

Example 2 (KS BH). The entropy for $\mathrm{KS} \mathrm{BH}$ [8] should read

$$
\begin{aligned}
\mathcal{S}_{0, \pm} & =\pi r_{ \pm}^{2}, \\
r_{ \pm} & =M \pm \sqrt{M^{2}-\frac{1}{2 \omega}},
\end{aligned}
$$

where $\omega$ is coupling constant and $r_{+}$and $r_{-}$are $\mathrm{EH}$ and $\mathrm{CH}$, respectively. The Hawking temperature becomes

$$
T_{ \pm}=\frac{\omega\left(r_{ \pm}-r_{\mp}\right)}{4 \pi\left(1+\omega r_{ \pm}^{2}\right)} .
$$

Therefore, the entropy correction for $\mathrm{KS} \mathrm{BH}$ is given by

$$
\mathcal{S}_{ \pm}=\mathcal{S}_{0, \pm}-\frac{1}{2} \ln \left|\frac{\omega^{2} r_{ \pm}^{2}\left(r_{ \pm}-r_{\mp}\right)^{2}}{16 \pi\left(1+\omega r_{ \pm}^{2}\right)}\right|+\cdots
$$

It implies that when the logarithmic correction is taken into consideration the entropy product is not mass-independent (universal).

Now we take the AdS space. First, it should be Schwarzschild-AdS space-time (in the limit $\ell \rightarrow \infty$, one gets the Schwarzschild $\mathrm{BH}$. Here the horizon is at $r_{+}=2 M$ and $\mathcal{S}_{0,+}=4 \pi M^{2}$. Thus, one obtains the logarithmic correction as $\mathcal{S}_{+}=4 \pi M^{2}+(1 / 2) \ln |16 \pi|$. Therefore, it indicates that the logarithmic correction must be mass dependent. Therefore, it is not universal).

Example 3 (Schwarzschild-AdS BH). The only physical horizon [4] is at

$$
r_{+}=\frac{2 \ell}{\sqrt{3}} \sinh \left[\frac{1}{3} \sinh ^{-1}\left(3 \sqrt{3} \frac{M}{\ell}\right)\right] .
$$

Thus, the entropy of $\mathscr{H}^{+}$should be

$$
\mathcal{\delta}_{0,+}=\pi r_{+}^{2},
$$

where $\Lambda=-3 / \ell^{2}$ is cosmological constant. The $\mathrm{BH}$ temperature reads

$$
T_{+}=\frac{1}{4 \pi r_{+}}\left(1+3 \frac{r_{+}^{2}}{\ell^{2}}\right) .
$$

Now the entropy correction formula should read

$$
\mathcal{S}_{+}=\mathcal{S}_{0,+}-\frac{1}{2} \ln \left|\frac{\left(1+3\left(r_{+}^{2} / \ell^{2}\right)\right)^{2}}{16 \pi}\right|+\cdots .
$$

In fact, in both cases, with logarithmic correction and without logarithmic correction, the entropy depends on the mass parameter; thus, it is not universal and therefore it is not quantized.

Now we take the RN-AdS case [4].

Example 4 (RN-AdS BH). The quartic Killing horizon equation becomes

$$
r^{4}+\ell^{2} r^{2}-2 M \ell^{2} r+Q^{2} \ell^{2}=0 .
$$

There are at least two real zeros which correspond to two physical horizons, namely, $\mathrm{EH}, r_{+}$, and $\mathrm{CH}, r_{-}$.

The entropy should read

$$
\mathcal{S}_{0, \pm}=\pi r_{ \pm}^{2} .
$$

The $\mathrm{BH}$ temperature of $\mathscr{H}^{ \pm}$is given by

$$
T_{ \pm}=\frac{1}{4 \pi r_{ \pm}}\left(3 \frac{r_{ \pm}^{2}}{\ell^{2}}-\frac{Q^{2}}{r_{ \pm}^{2}}+1\right) .
$$

Therefore, the logarithmic correction becomes

$$
\mathcal{S}_{ \pm}=\mathcal{S}_{0, \pm}-\frac{1}{2} \ln \left|\frac{\left(3\left(r_{+}^{2} / \ell^{2}\right)-Q^{2} / r_{+}^{2}+1\right)^{2}}{16 \pi}\right|+\cdots
$$

It implies that the product of logarithmic correction is not mass-independent.

Now we take the spinning $\mathrm{BH}$.

Example 5 (Kerr $\mathrm{BH})$. The $\mathrm{BH}$ entropy and $\mathrm{BH}$ temperature [15] are

$$
\begin{gathered}
\mathcal{S}_{0, \pm}=\pi\left(r_{ \pm}^{2}+a^{2}\right), \\
T_{ \pm}=\frac{r_{ \pm}-r_{\mp}}{4 \pi\left(r_{ \pm}^{2}+a^{2}\right)},
\end{gathered}
$$

where $r_{ \pm}=M \pm \sqrt{M^{2}-a^{2}}, M$, and $a=J / M$ are mass and spin parameter of the $\mathrm{BH}$, respectively.

Now the logarithmic correction is computed to be

$$
\mathcal{S}_{ \pm}=\mathcal{S}_{0, \pm}-\frac{1}{2} \ln \left|\frac{\left(r_{ \pm}-r_{\mp}\right)^{2}}{16 \pi\left(r_{ \pm}^{2}+a^{2}\right)}\right|+\cdots .
$$

It also indicates that whenever we take the logarithmic correction; the entropy product of $\mathscr{H}^{ \pm}$is not universal.

Next we take charged rotating $\mathrm{BH}$. 
Example 6 (Kerr-Newman $\mathrm{BH})$. The $\mathrm{BH}$ entropy and $\mathrm{BH}$ temperature [15] should read

$$
\begin{gathered}
\mathcal{S}_{0, \pm}=\pi\left(r_{ \pm}^{2}+a^{2}\right), \\
T_{ \pm}=\frac{r_{ \pm}-r_{\mp}}{4 \pi\left(r_{ \pm}^{2}+a^{2}\right)},
\end{gathered}
$$

where $r_{ \pm}=M \pm \sqrt{M^{2}-a^{2}-Q^{2}}$ and $M, a$, and $Q$ correspond to the mass, the spin parameter, and the charge of $\mathrm{BH}$, respectively.

The logarithmic correction is derived to be

$$
\mathcal{S}_{ \pm}=\mathcal{S}_{0, \pm}-\frac{1}{2} \ln \left|\frac{\left(r_{ \pm}-r_{\mp}\right)^{2}}{16 \pi\left(r_{ \pm}^{2}+a^{2}\right)}\right|+\cdots
$$

Again we observe that when we take logarithmic correction the entropy product of $\mathscr{H}^{ \pm}$for Kerr-Newman $\mathrm{BH}[1,6]$ is not mass-independent.

Example 7 (Kerr-Newman AdS BH). The horizon equation [33] is given by

$$
\Delta_{r}=\left(r^{2}+a^{2}\right)\left(1+\frac{r^{2}}{\ell^{2}}\right)-2 M r+Q_{e}^{2}+Q_{m}^{2}=0
$$

which implies that the quartic order horizon equation

$$
r^{4}+\left(\ell^{2}+a^{2}\right) r^{2}-2 M \ell^{2} r+\left(a^{2}+Q_{e}^{2}+Q_{m}^{2}\right) \ell^{2}=0
$$

This equation has two real zeros which correspond to two physical horizons, namely, $r_{ \pm}$, where $Q_{e}$ and $Q_{m}$ are electric and magnetic charge parameters, respectively. The $\mathrm{BH}$ entropy and $\mathrm{BH}$ temperature are

$$
\begin{aligned}
& \mathcal{S}_{0, \pm}=\frac{\pi\left(r_{ \pm}^{2}+a^{2}\right)}{\left(1-a^{2} / \ell^{2}\right)} \\
& T_{ \pm} \\
& =\frac{r_{ \pm}\left(1+a^{2} / \ell^{2}+3\left(r_{ \pm}^{2} / \ell^{2}\right)-\left(a^{2}+Q_{e}^{2}+Q_{m}^{2}\right) / r_{ \pm}^{2}\right)}{4 \pi\left(r_{ \pm}^{2}+a^{2}\right)} .
\end{aligned}
$$

The logarithmic correction for KN-AdS BH should read

$$
\begin{aligned}
\mathcal{S}_{ \pm} & =\mathcal{S}_{0, \pm}-\frac{1}{2} \\
& \cdot \ln \left|\frac{r_{ \pm}^{2}\left(1+a^{2} / \ell^{2}+3\left(r_{ \pm}^{2} / \ell^{2}\right)-\left(a^{2}+Q_{e}^{2}+Q_{m}^{2}\right) / r_{ \pm}^{2}\right)^{2}}{16 \pi\left(1-a^{2} / \ell^{2}\right)\left(r_{ \pm}^{2}+a^{2}\right)}\right| \\
& +\cdots
\end{aligned}
$$

Example 8 (nonrotating BTZ BH). The $\mathrm{BH}$ horizon is at $r_{+}=$ $\sqrt{8 G_{3} M \ell} \cdot G_{3}$ is $3 \mathrm{D}$ Newtonian constant. The $\mathrm{BH}$ entropy of $\mathscr{H}^{+}$for BTZ BH is

$$
\mathcal{S}_{0,+}=\frac{2 \pi r_{+}}{4 G_{3}}
$$

The $\mathrm{BH}$ temperature is

$$
T_{+}=\frac{r_{+}}{2 \pi \ell^{2}}
$$

where $\Lambda=-3 / \ell^{2}$ is cosmological constant. Thus, the $\mathrm{BH}$ entropy correction for $\mathrm{BTZ} \mathrm{BH}$ is

$$
\mathcal{S}_{+}=\mathcal{S}_{0,+}-\frac{1}{2} \ln \left|\frac{r_{+}^{3}}{8 \pi G_{3} \ell^{4}}\right|+\cdots .
$$

In fact, it is isolated case and there is only one horizon; therefore, both the logarithmic correction of entropy and without logarithmic corrections term are mass dependent.

Example 9 (rotating $\mathrm{BTZ} \mathrm{BH}$ ). The $\mathrm{BH}$ horizons for rotating $\mathrm{BTZ} \mathrm{BH}[34,35]$ are given by

$$
r_{ \pm}=\sqrt{4 G_{3} \mathscr{M} \ell^{2}\left(1 \pm \sqrt{1-\frac{J^{2}}{M^{2} \ell^{2}}}\right)}
$$

The $\mathrm{BH}$ entropy of $\mathscr{H}^{ \pm}$is

$$
\mathcal{S}_{0, \pm}=\frac{2 \pi r_{ \pm}}{4 G_{3}}
$$

and the Hawking temperature of $\mathscr{H}^{ \pm}$should read

$$
T_{ \pm}=\frac{r_{ \pm}^{2}-r_{\mp}^{2}}{2 \pi \ell^{2} r_{ \pm}}
$$

Therefore, the $\mathrm{BH}$ entropy correction is calculated to be

$$
\mathcal{S}_{ \pm}=\mathcal{S}_{0, \pm}-\frac{1}{2} \ln \left|\frac{\left(r_{ \pm}^{2}-r_{\mp}^{2}\right)^{2}}{8 \pi G_{3} \ell^{4} r_{ \pm}}\right|+\cdots .
$$

It is clear from the calculation that the product depends on the mass parameter.

Example 10 (charged dilaton $\mathrm{BH}$ ). The horizons of charged dilation $\mathrm{BH}[36]$ are at

$$
\begin{aligned}
& r_{+}=M+\sqrt{M^{2}-\left(\frac{2 n}{1+n}\right) Q^{2}}, \\
& r_{-}=\frac{1}{n}\left[M+\sqrt{M^{2}-\left(\frac{2 n}{1+n}\right) Q^{2}}\right],
\end{aligned}
$$

where $n$ is given by

$$
n=\frac{1-a^{2}}{1+a^{2}} .
$$

The $\mathrm{BH}$ temperature of $\mathscr{H}^{ \pm}$reads

$$
\begin{aligned}
& T_{+}=\frac{1}{4 \pi r_{+}}\left(\frac{r_{+}-r_{-}}{r_{+}}\right)^{n}, \\
& T_{-}=0 .
\end{aligned}
$$


The entropy for both the horizons is

$$
\begin{aligned}
& \mathcal{S}_{0,+}=\pi r_{+}^{2}\left(\frac{r_{+}-r_{-}}{r_{+}}\right)^{1-n}, \\
& \mathcal{S}_{0,-}=0 .
\end{aligned}
$$

Thus, the $\mathrm{BH}$ entropy correction for $\mathscr{H}^{+}$should be

$$
\mathcal{S}_{+}=\mathcal{S}_{0,+}-\frac{1}{2} \ln \left|\frac{\left(\left(r_{+}-r_{-}\right) / r_{+}\right)^{1+n}}{16 \pi}\right|+\cdots .
$$

And the entropy correction for $\mathscr{H}^{-}$is

$$
\mathcal{S}_{+}=0 \text {. }
$$

This is an interesting case because the entropy product of $\mathscr{H}^{ \pm}$ and the entropy product with logarithmic correction both go to zero value. The logarithmic correction survives for $\mathscr{H}^{+}$ and only when we have taken into account the logarithmic correction for $\mathscr{H}^{-}$it breaks down and therefore the product also breaks down.

Example 11 (Kerr-Sen BH). The horizon for Kerr-Sen [37, 38] $\mathrm{BH}$ is situated at

$$
r_{ \pm}=\left(M-\frac{Q^{2}}{2 M}\right) \pm \sqrt{\left(M-\frac{Q^{2}}{2 M}\right)^{2}-a^{2}}
$$

The $\mathrm{BH}$ entropy and $\mathrm{BH}$ temperature for Sen $\mathrm{BH}$ are

$$
\begin{gathered}
\mathcal{S}_{0, \pm}=2 \pi M r_{ \pm} \\
T_{ \pm}=\frac{r_{ \pm}-r_{\mp}}{8 \pi M r_{ \pm}} .
\end{gathered}
$$

Therefore, the logarithmic correction is calculated to be

$$
\mathcal{S}_{ \pm}=\mathcal{S}_{0, \pm}-\frac{1}{2} \ln \left|\frac{\left(r_{ \pm}-r_{\mp}\right)^{2}}{32 \pi M r_{ \pm}}\right|+\cdots .
$$

Example 12 (Sultana-Dyer $\mathrm{BH}$ ). This is an example of a dynamical cosmological $\mathrm{BH}$ [39]. The horizon is located at $r_{+}=2 m$, where $m$ is the mass of $\mathrm{BH}$.

The entropy (the surface area at $t=0$ indicates that the cosmological $\mathrm{BH}$ is formed initially from Big-Bang singularity. $t^{4}$ is the conformal factor) and temperature of this $\mathrm{BH}$ are

$$
\begin{gathered}
\mathcal{S}_{0,+}=\frac{\mathscr{A}_{+}}{4}=\pi t^{4} r_{+} \\
T_{+}=\frac{1}{4 \pi t^{2} r_{+}} .
\end{gathered}
$$

The logarithmic correction is found to be

$$
\mathcal{S}_{+}=\mathcal{S}_{0,+}-\frac{1}{2} \ln \left|\frac{1}{16 \pi}\right|+\cdots .
$$

The interesting fact in this case is that we have found that the entropy correction term in the logarithmic correction term is mass-independent, whereas without logarithmic term it is mass-dependent.
Example 13 (charged BHs in $f(R)$ gravity). The $f(R)$ gravity $[40,41]$ is interesting because it is equivalent to Einstein gravity coupled to matter, where $f(R)$ is an arbitrary function of the scalar curvature. The horizon function at the constant scalar curvature $R=R_{0}$ is given by

$$
\mathcal{N}(r)=1-\frac{2 \mu}{r}+\frac{q^{2}}{\alpha r^{2}}-\frac{R_{0}}{12} r^{2}=0,
$$

where $\alpha=1+f^{\prime}\left(R_{0}\right)$. The quantities $\mu$ and $q$ are related to $M$ (ADM mass) and $Q$ (electric charge) which are

$$
\begin{aligned}
& M=\mu \alpha, \\
& Q=\frac{q}{\sqrt{2 \alpha}} .
\end{aligned}
$$

The entropy for all the horizons is

$$
\mathcal{S}_{0, i}=\pi \alpha r_{i}^{2}
$$

and the $\mathrm{BH}$ temperature should read

$$
T_{i}=\frac{1}{4 \pi r_{i}}\left(1-\frac{q^{2}}{\alpha r_{i}^{2}}-\frac{R_{0}}{4} r_{i}^{2}\right)
$$

The logarithmic correction of entropy becomes

$$
\mathcal{S}_{i}=\mathcal{S}_{0, i}-\frac{1}{2} \ln \left|\frac{\alpha\left(1-q^{2} / \alpha r_{i}^{2}-\left(R_{0} / 4\right) r_{i}^{2}\right)^{2}}{16 \pi r_{i}^{2}}\right|+\cdots
$$

Example 14 (5D Gauss-Bonnet $\mathrm{BH}$ ). The horizon radii for 5D Gauss-Bonnet BH [41] are located at

$$
r_{ \pm}=\frac{1}{\sqrt{2}} \sqrt{\left(2 \mu-\alpha_{d}\right) \pm \sqrt{\left(2 \mu-\alpha_{d}\right)^{2}-4 q^{2}}}
$$

where $\mu=4 M / 3 \pi$ and $q=(4 / \pi)^{2 / 3} \mathrm{Q}$.

The entropy of $\mathscr{H}^{ \pm}$is

$$
\mathcal{S}_{0, \pm}=\frac{\pi^{2} r_{ \pm}^{3}}{2}\left(1+\frac{6 \alpha_{d}}{r_{ \pm}^{2}}\right) .
$$

The $\mathrm{BH}$ temperature of $\mathscr{H}^{ \pm}$reads

$$
T_{ \pm}=\frac{r_{ \pm}^{2}-r_{\mp}^{2}}{2 \pi r_{ \pm}\left(r_{ \pm}^{2}+2 \alpha_{d}\right)}
$$

where $\alpha_{d}=(d-3)(d-4) \alpha=2 \alpha$ and $\alpha_{d}$ is Gauss-Bonnet coupling constant.

The logarithmic correction of entropy $\mathscr{H}^{ \pm}$becomes

$$
\mathcal{S}_{ \pm}=\mathcal{S}_{0, \pm}-\frac{1}{2} \ln \left|\frac{r_{ \pm}\left(1+6 \alpha_{d} / r_{ \pm}^{2}\right)\left(r_{ \pm}^{2}-r_{\mp}^{2}\right)^{2}}{8\left(r_{ \pm}^{2}+2 \alpha_{d}\right)^{2}}\right|+\cdots
$$

It follows from the several examples that when the logarithmic correction is considered the entropy product formula is not mass-independent (universal) and therefore it is not quantized. 
To sum up, we computed the general logarithmic corrections to the $\mathrm{BH}$ entropy product formula of inner horizon and outer horizon by taking into consideration the effects of statistical quantum fluctuations around the thermal equilibrium and also via CFT. We showed, followed by our earlier work [11], that whenever we take the first-order logarithmic correction to the entropy product formula, it is not universal and also it cannot be quantized. What is new in this work is that when we have chosen the exact entropy function followed by CFT and by taking the effects of quantum fluctuations, the logarithmic correction formula of $\mathscr{H}^{ \pm}$should depend solely on the value of $\mathrm{BH}$ temperature of $\mathscr{H}^{ \pm}$and $\mathrm{BH}$ entropy of $\mathscr{H}^{ \pm}$at the thermal equilibrium.

\section{Conflicts of Interest}

The author declares that they have no conflicts of interest.

\section{References}

[1] M. Ansorg and J. Hennig, "Inner Cauchy horizon of axisymmetric and stationary black holes with surrounding matter in Einstein-Maxwell theory," Physical Review Letters, vol. 102, Article ID 221102, 2009.

[2] M. Cvetič et al., "Universal area product formulae for rotating and charged black holes in four and higher dimensions," Physical Review Letters, vol. 106, Article ID 121301, 2011.

[3] A. Castro and M. J. Rodriguez, "Universal properties and the first law of black hole inner mechanics," Physical Review D, vol. 86, Article ID 024008, 2012.

[4] M. Visser, "Area products for stationary black hole horizons," Physical Review D, vol. 88, Article ID 044014, 2013.

[5] V. Faraoni and A. F. Z. Moreno, "Are quantization rules for horizon areas universal?” Physical Review D, vol. 88, Article ID 044011, 2013.

[6] P. Pradhan, "Black hole interior mass formula," The European Physical Journal C, vol. 74, p. 2887, 2014.

[7] J. Hennig, "Geometric relations for rotating and charged AdS black holes," Classical and Quantum Gravity, vol. 31, no. 13, Article ID 135005, 9 pages, 2014.

[8] P. Pradhan, "Thermodynamic product formula for HořavaLifshitz black hole," Physics Letters B, vol. 747, pp. 64-67, 2015.

[9] P. Pradhan, "Area (or entropy) product formula for a regular black hole," General Relativity and Gravitation, vol. 48, no. 2, p. 19, 2016.

[10] F. Larsen, "String model of black hole microstates," Physical Review D, vol. 56, no. 2, pp. 1005-1008, 1997.

[11] P. Pradhan, "Logarithmic corrections in black hole entropy product formula," General Relativity and Gravitation, vol. 48, no. 7, p. 98, 2016.

[12] J. D. Bekenstein, "Black holes and the second law," Lettere Al Nuovo Cimento 2, vol. 4, no. 15, pp. 737-740, 1972.

[13] J. D. Bekenstein, "Black holes and entropy," Physical Review. D. Particles and Fields. Third Series, vol. 7, pp. 2333-2346, 1973.

[14] J. M. Bardeen, B. Carter, and S. W. Hawking, "The four laws of black hole mechanics," Communications in Mathematical Physics, vol. 31, pp. 161-170, 1973.

[15] P. C. W. Davies, “Thermodynamics of black holes," Reports on Progress in Physics, vol. 41, no. 8, p. 1313, 1978.
[16] Arnold Sommerfeld, Thermodynamics and Statistical Mechanics, Academic Press, New York, 1956.

[17] A. Strominger and C. Vafa, "Microscopic origin of the Bekenstein-Hawking entropy," Physics Letters B, vol. 379, no. 1-4, pp. 99-104, 1996.

[18] A. Ashtekar, J. Baez, A. Corichi, and K. Krasnov, "Quantum geometry and black hole entropy," Physical Review Letters, vol. 80, no. 5, pp. 904-907, 1998.

[19] S. Carlip, "Entropy from conformal field theory at Killing horizons," Classical and Quantum Gravity, vol. 16, no. 10, pp. 3327-3348, 1999.

[20] S. Carlip, "Logarithmic corrections to black hole entropy, from the Cardy formula," Classical and Quantum Gravity, vol. 17, no. 20, pp. 4175-4186, 2000.

[21] R. K. Kaul and P. Majumdar, "Quantum black hole entropy," Physics Letters. B, vol. 439, no. 3-4, pp. 267-270, 1998.

[22] R. K. Kaul and P. Majumdar, "Logarithmic correction to the Bekenstein-Hawking entropy," Physical Review Letters, vol. 84, no. 23, pp. 5255-5257, 2000.

[23] S. Das et al., "A new holographic entropy bound from quantum geometry," Physical Review D, vol. 63, Article ID 044019, 2001.

[24] S. Das, P. Majumdar, and R. K. Bhaduri, "General logarithmic corrections to black-hole entropy," Classical and Quantum Gravity, vol. 19, no. 9, p. 2355, 2002.

[25] M. Guica, T. Hartman, W. Song, and A. Strominger, "The Kerr/CFT correspondence," Physical Review D, vol. 80, no. 12, 9 pages, 2009.

[26] A. Sen, "Microscopic and macroscopic entropy of extremal black holes in string theory," General Relativity and Gravitation, vol. 46, no. 5, pp. 1-12, 2014.

[27] A. Bohr and B. R. Mottelson, Nuclear Structure, vol. 1, pp. 281283, 1969.

[28] R. K. Bhaduri, in Models of the Nucleon, p. 116, Addison-Wesley, 1988.

[29] A. Kehagias and K. Sfetsos, "The black hole and FRW geometries of non-relativistic gravity," Physics Letters B, vol. 678, no. 1, pp. 123-126, 2009.

[30] P. Hořava, "Spectral dimension of the universe in quantum gravity at a Lifshitz point," Physical Review Letters, vol. 102, no. 16, Article ID 161301, 2009.

[31] P. Horava, “Quantum gravity at a Lifshitz point," Physical Review $D$, vol. 79, no. 8, 15 pages, 2009.

[32] P. Horava, "Membranes at quantum criticality," Journal of High Energy Physics, vol. 0903, no. 020, 2009.

[33] M. M. Caldarelli, G. Cognola, and D. Klemm, "Thermodynamics of Kerr-Newman-AdS black holes and conformal field theories," Classical and Quantum Gravity, vol. 17, no. 2, pp. 399420, 2000 .

[34] M. Bañados et al., "The black hole in three-dimensional spacetime," Physical Review Letters, vol. 69, p. 1849, 1992.

[35] P. Pradhan, "Entropy product formula for a spinning BTZ black hole," JETP Letters, vol. 102, no. 7, article 427, 2015.

[36] B. Majeed et al., "Thermodynamic relations for kiselev and dilaton black hole," Advances in High Energy Physics, vol. 2015, article 124910, Article ID 124910, 2015.

[37] A. Sen, "Rotating charged black hole solution in heterotic string theory," Physical Review Letters, vol. 69, no. 7, pp. 1006-1009, 1992.

[38] P. Pradhan, "Thermodynamic products for Sen black hole," The European Physical Journal C, vol. 76, p. 31, 2016. 
[39] J. Sultana and C. C. Dyer, "Cosmological black holes: A black hole in the Einstein-de Sitter universe," General Relativity and Gravitation, vol. 37, no. 8, pp. 1347-1370, 2005.

[40] T. Moon, Y. S. Myung, and E. J. Son, “ $f(R)$ black holes," General Relativity and Gravitation, vol. 43, no. 11, p. 3079, 2011.

[41] A. Castro et al., "On the universality of inner black hole mechanics and higher curvature gravity, Journal of High Energy Physics, vol. 07, no. 164, 2013. 

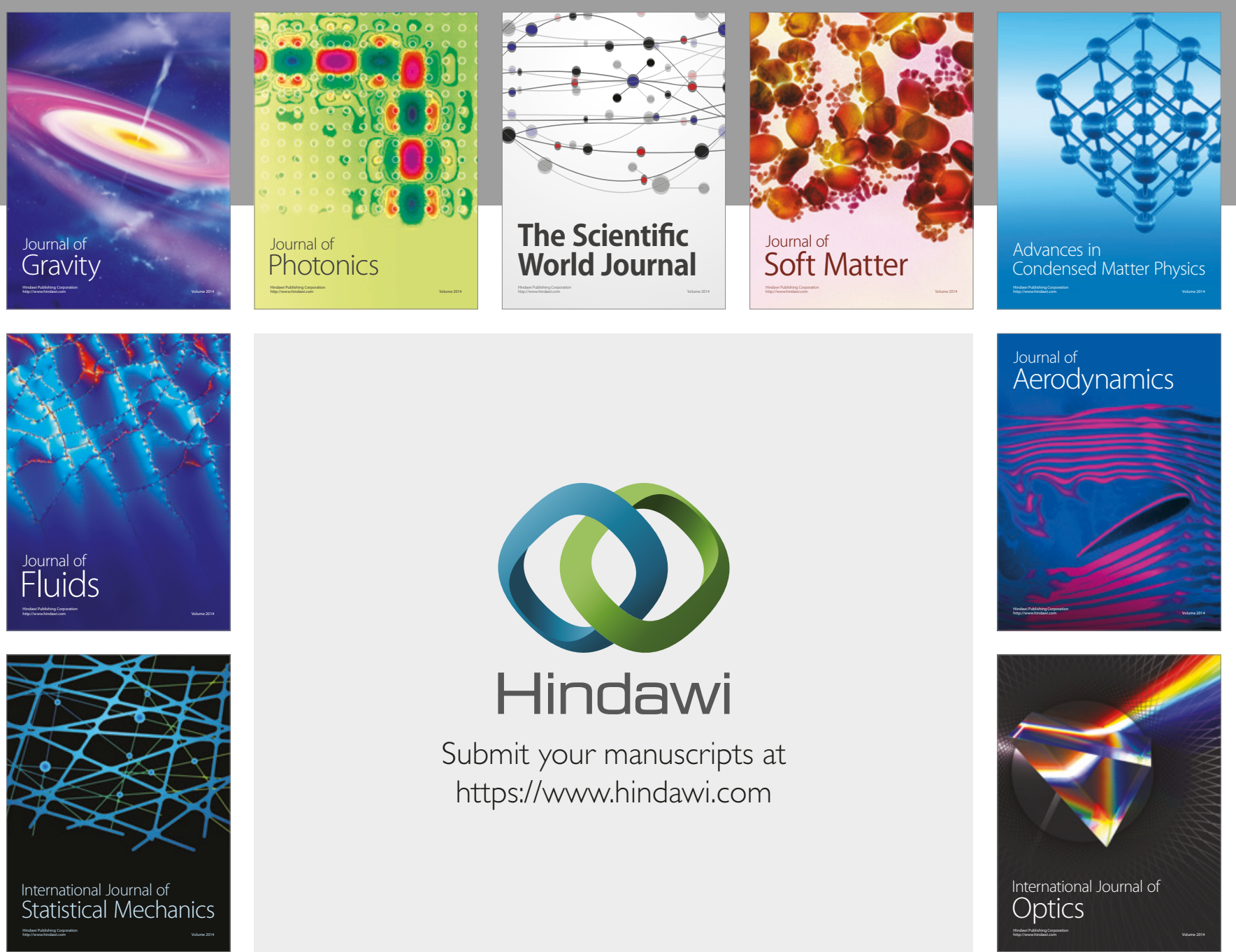

Submit your manuscripts at

https://www.hindawi.com
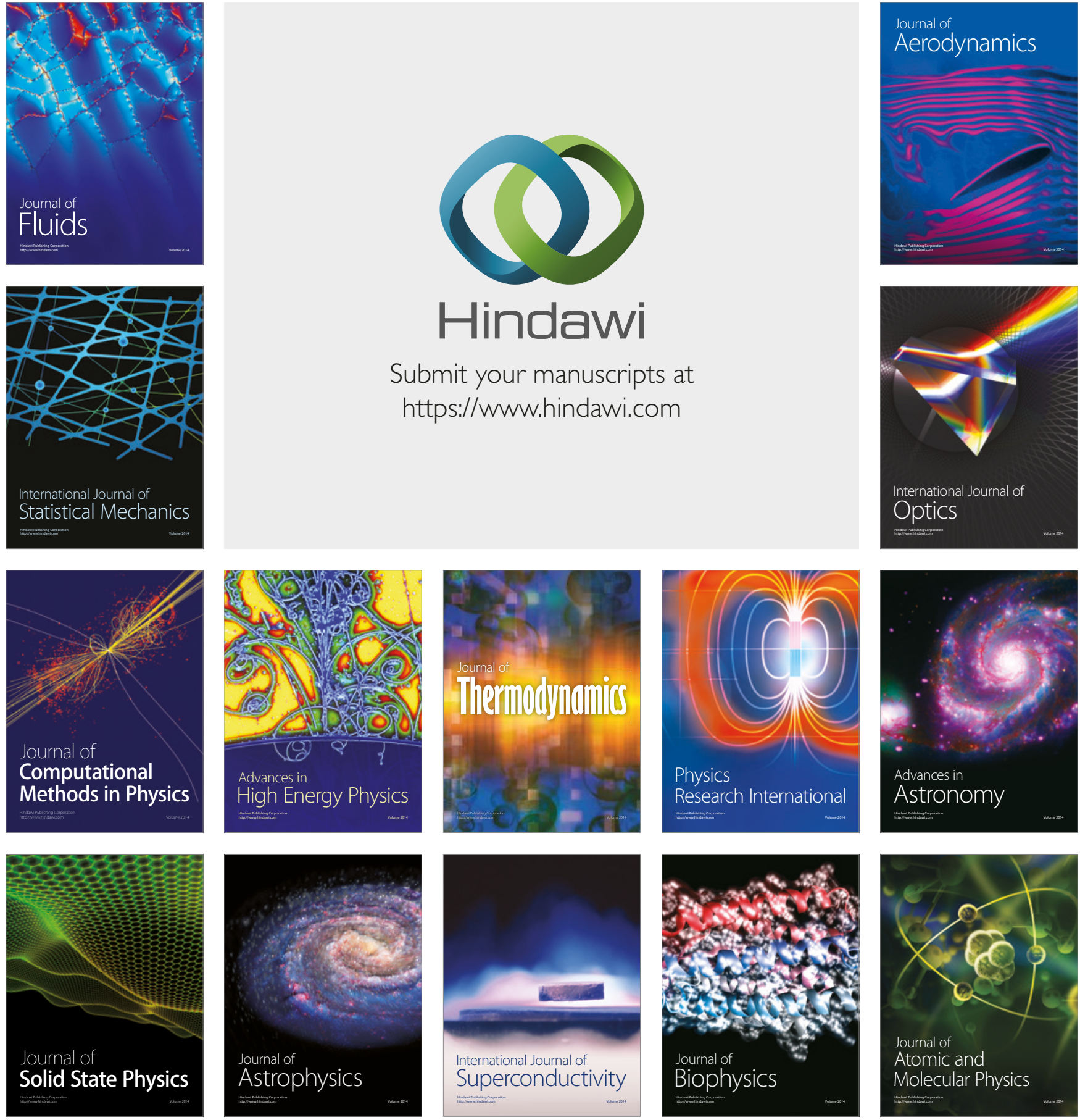